\title{
Giant Lipomatosis of the Sciatic Nerve: Unique Magnetic Resonance Imaging Findings
}

\author{
Ali Firat Sarp, ${ }^{1,}$ and Yeliz Pekcevik ${ }^{2}$ \\ ${ }^{1}$ Radiology Department, Ataturk Training and Research Hospital, Izmir Katip Celebi University, Izmir, Turkey \\ ${ }^{2}$ Radiology Department, Tepecik Training and Research Hospital, Izmir, Turkey \\ "Corresponding author: Ali Firat Sarp, Radiology Department, Ataturk Training and Research Hospital, Izmir Katip Celebi University, Izmir, Turkey. Fax: +90-2322431530, E-mail: \\ firat_sarp@yahoo.com
}

Received 2014 June 30; Revised 2014 August 07; Accepted 2014 October 07.

\begin{abstract}
Lipomatosis of the nerve, also known as fibrolipomatous hamartoma, is characterized by the infiltration of the nerve by fibro-fatty tissue. The affected nerve becomes thicker, and it simulates a mass lesion. Lipomatosis usually affects the median nerve and lipomatosis of the sciatic nerve is extremely rare. Magnetic resonance imaging (MRI) is the key to diagnosis, and it is usually pathognomonic. In this report, MRI and diffusion-weighted MRI findings of a case of a giant sciatic nerve lipomatosis without macrodactyly are presented. The MRI findings are unique, and awareness of the MRI features of this rare soft tissue mass may prevent unnecessary biopsies and surgeries.
\end{abstract}

Keywords: Sciatic Nerve, Lipomatosis, Magnetic Resonance Imaging, Diffusion Magnetic Resonance Imaging

\section{Introduction}

Lipomatosis of the nerve was first described in 1953 by Mason (1). It was previously known as neural fibrolipoma, intraneural lipoma, perineural lipoma, or fibrolipomatous hamartoma (2). In 2002, the world health organization (WHO) suggested the term lipomatosis of the nerve for this entity (3). Lipomatosis of the nerve is characterized by an abnormal infiltration of the nerve by fatty and fibrous tissue; thus, the affected nerve becomes thicker and simulates a mass lesion. It may be associated with macrodactyly, sometimes enough to cause localized gigantism (1, $4,5)$. Although most of the cases present in youth, it may be seen in any age (5). Lipomatosis usually affects the nerves of the upper extremities, especially median nerves. The involvement of nerves of the lower extremity is extremely rare (2). Based on our literature review, only few cases with or without macrodactyly have been reported (4-6).

Although both ultrasound and computerized tomography are useful in diagnosis, magnetic resonance imaging (MRI) with high soft tissue resolution is the gold standard. Lipomatosis of the sciatic nerve has unique MRI findings that may prevent unnecessary, contraindicated biopsies. MRI provides the diagnosis of lipomatosis of the nerve with confidence, without requiring histopathologic correlation $(2,6)$. In this report, the MRI and diffusion-weighted MRI findings of a case of giant sciatic nerve lipomatosis are presented.

\section{Case Presentation}

A 60-year-old female patient was referred to this clinic to obtain a contrast-enhanced MRI with a prediagnosis of palpable posterior thigh mass and buttock pain. Her chief complaint was swelling in the posterior aspect of the distal left thigh that became larger in the last few years. Contrast-enhanced MRI and diffusion-weighted imaging (DWI), which are part of the tumor protocol, were performed. Axial, coronal T1-weighted, fat-suppressed T2weighted, and contrast-enhanced fat-suppressed axial and coronal T1-weighted images were obtained using a 1.5-Tesla system (Achieva; Philips, the Netherlands) (Figure 1).

The DWI was obtained before contrast administration with a single-shot spin-echo echo-planar imaging (EPI) technique (repetition time, $4500 \mathrm{~ms}$; echo time, $105 \mathrm{~ms}$; directions of the motion-probing gradients, three orthogonal axes; b value, 0 and 1000 seconds $/ \mathrm{mm}^{2}$; field of view, $220 \mathrm{~mm}$; matrix size, $128 \times 80$-128; section thickness, $5 \mathrm{~mm}$ with $0.2-1.0-\mathrm{mm}$ intersection gaps; NSA and SENSE with a reduction factor of 1-1.5). A $7.5 \times 25 \mathrm{~cm}$, well-circumscribed giant mass that originated from the osseous pelvis and extended to the gluteal region was detected that located in the sciatic nerve course. Its intensity was similar to those of subcutaneous fat on all pulse sequences. There was a fine fibrillar appearance inside of it. There was no deformity or destruction of the adjacent osseous structures. The mass showed no enhancement after contrast administration.

On the DWI and apparent diffusion coefficient (ADC) 

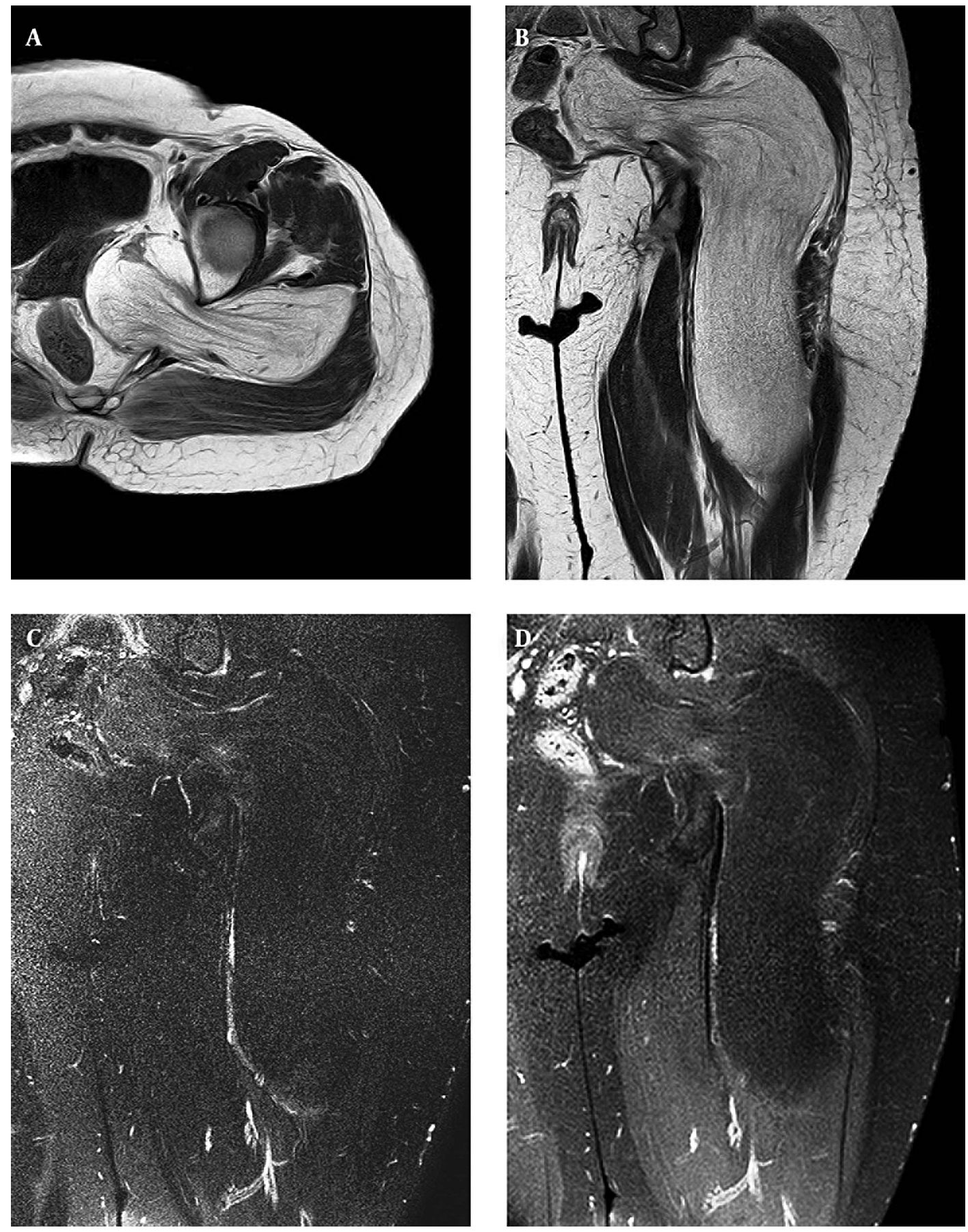

Figure 1. A 60-year-old female patient with a palpable posterior thigh mass and buttock pain. T1-weighted axial (A) and coronal (B) images show a giant space-occupying lesion that is located in the sciatic nerve course. The tumor is isointense to the subcutaneous fat and there is fine fibrillar appearance inside of it. No signal increase on fat-suppressed T2-weighted image is seen (C). Postcontrast fat-suppressed T1-weighted image (D) shows no intensive enhancement except for faint signal increase at the fibrillar appearance that may represent inherent neural fiber enhancement.

mapping, the lesion demonstrated a low signal, isointense to subcutaneous fat, due to a fat-saturated pulse that was used to exclude chemical-shift artifacts. The typical MRI findings and the sciatic nerve course of the lesion allowed 
the diagnosis of lipomatosis of the sciatic nerve (Figure 2). Since the patient had suffered an increasing limitation of movement caused by the giant, space-occupying lesion, an internal neurolysis was performed with microsurgical techniques.

\section{Discussion}

The MRI and diffusion-weighted MRI findings of a case with giant sciatic nerve lipomatosis were presented. The involvement of nerves of the lower extremity is extremely rare, and, to the best of our knowledge, diffusion-weighted MRI findings have not been reported. The abnormal fatty tissue that surrounds the nerve fibers and fusiform enlargement of the nerve are seen as spaghetti-like in longitudinal section and as coaxial cable-like in cross-section; they are usually pathognomonic for lipomatosis of the nerve on T1-weighted images. T2-weighted images do not typically show an increased signal $(2,4,6)$. With fat suppression techniques, the high signal of the adipose tissue disappears almost completely. After an intravenous contrast injection, enhancement is not present. However, there may be faint enhancement due to the compression of surrounding structures. In a fusiform mass with these typical findings, the diagnosis is clear; there is no need for further diagnostic work up or surgical biopsy.

A conventional MRI helps characterize the lesions, but it provides low specificity in the differential diagnosis of soft tissue tumors because many of the lesions exhibit nonspecific characteristics. A DWI used in association with a conventional MRI improves the diagnostic confidence. A DWI allows quantitative and qualitative analyses of tissue cellularity and cell membrane integrity, and it has been widely used for tumor detection and characterization (7). It has been reported that a DWI can differentiate benign from malignant soft tissue tumors $(8,9)$. In this particular case, the described conventional MRI findings were characteristic for lipomatosis; although it is rare in this location with these dimensions. The MRI confirmed the diagnosis. The DWI, which is part of the routine protocol, did not add much information. While performing the DWI, in all of the images, a fat-saturated pulse was used to exclude the chemical-shift artifacts and to reduce the signal in this tumor, confirming its lipomatous nature.

The first case of sciatic nerve lipomatosis was found in Marom and Helms' study, which was published in 1999 (6). They reviewed the imaging characteristics of 10 cases of nerve lipomatosis in a retrospective study. In 1 of the 10 cases, a 75-year-old male had sciatic nerve lipomatosis without macrodactyly. The size of the tumor was reported to be $28 \times 15 \times 120$ millimeters. Wong et al. reported two cases of sciatic nerve lipomatosis in 2006 (4). The first case was a 68-year-old male with sciatic neuropathic symptoms. An MRI of the left thigh showed a fusiform enlargement of the sciatic nerve, which began at the sciatic notch and extended distally about $10 \mathrm{~cm}$; thus, an MRI diagnosis sciatic nerve lipomatosis without macrodactyly was made. The second case was a 34-year-old female with the diagnosis of sciatic nerve lipomatosis with macrodactyly. Fandridis et al. reported a 26-year-old patient who suffered from sciatic nerve symptoms in 2009 (5). They found a $12 \mathrm{~cm}$ sciatic nerve lipomatosis without macrodactyly that started just distal to the ischial tuberosity.

To the best of our knowledge, only these three cases of sciatic nerve lipomatosis without macrodactyly have been reported. This case is the fourth case; it has the largest dimensions and showed the most severe mass effect.

As a final statement about lipomatosis of the nerve, it should be understood that nerve biopsy is usually contraindicated, and it may cause motor and sensory deficits. Therefore, correct interpretation of the MRI is crucial for the diagnosis.

\section{Footnotes}

Authors' Contribution: The study concept and design, Ali Firat Sarp; analysis and interpretation of data, Ali Firat Sarp and Yeliz Pekcevik; drafting of the manuscript, Ali Firat Sarp and Yeliz Pekcevik; Critical revision of the manuscript: Yeliz Pekcevik; study supervision, Yeliz Pekcevik; literature search, Ali Firat Sarp; Guarantor, Ali Firat Sarp and Yeliz Pekcevik.

Financial Disclosure: There is nothing to disclose. 

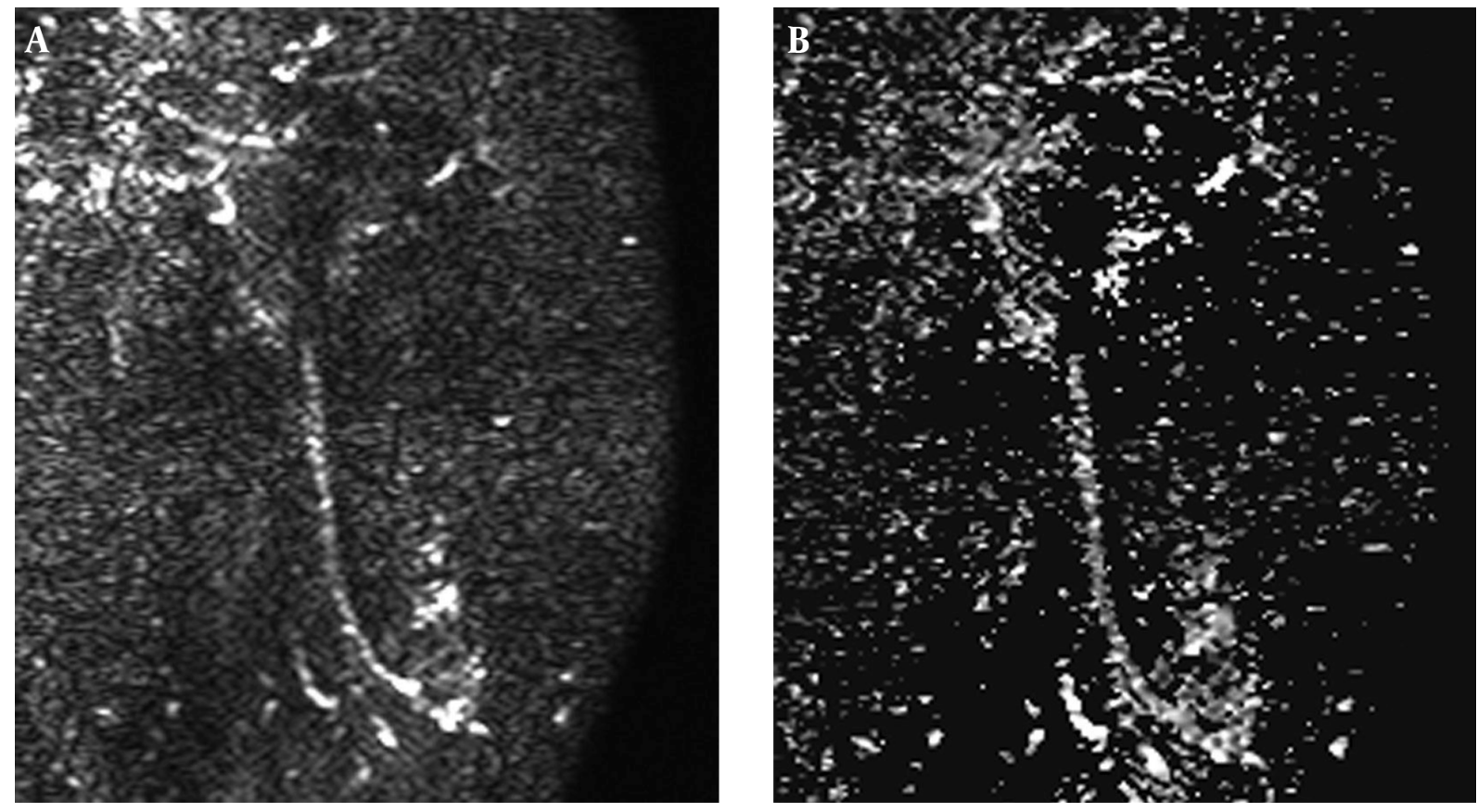

Figure 2. The lesion is isointense to the subcutaneous fat on diffusion weighted imaging (A) and apparent diffusion coefficient map (B).

\section{References}

1. Mason ML. Presentation of cases, proceedings of the american society of surgery of the hand.J Bone Joint Surg Am. 1953;35(1):273-5.

2. Murphey MD, Carroll JF, Flemming DJ, Pope TL, Gannon FH, Kransdorf MJ. From the archives of the AFIP: benign musculoskeletal lipomatous lesions. Radiographics. 2004;24(5):1433-66. doi: 10.1148/rg.245045120. [PubMed: 15371618].

3. Fletcher C, Unni KK. Pathology and genetics of tumours of soft tissue and bone. 4. Iarc; 2002.

4. Wong BZ, Amrami KK, Wenger DE, Dyck PJ, Scheithauer BW, Spinner RJ. Lipomatosis of the sciatic nerve: typical and atypical MRI features. Skeletal Radiol. 2006;35(3):180-4. doi: 10.1007/s00256-005-00348. [PubMed: 16283177$]$.

5. Fandridis EM, Kiriako AS, Spyridonos SG, Delibasis GE, Bourlos DN, Gerostathopoulos NE. Lipomatosis of the sciatic nerve: report of a case and review of the literature. Microsurgery. 2009;29(1):66-71. doi: 10.1002/micr.20571. [PubMed: 18942654].

6. Marom EM, Helms CA. Fibrolipomatous hamartoma: pathognomonic on MR imaging. Skeletal Radiol. 1999;28(5):260-4. [PubMed: 10424331].

7. Costa FM, Ferreira EC, Vianna EM. Diffusion-weighted magnetic resonance imaging for the evaluation of musculoskeletal tumors. Magn Reson Imaging Clin NAm. 2011;19(1):159-80. doi:10.1016/j.mric.2010.10.007. [PubMed: 21129640].

8. van Rijswijk CS, Kunz P, Hogendoorn PC, Taminiau AH, Doornbos J, Bloem JL. Diffusion-weighted MRI in the characterization of soft-tissue tumors. J Magn Reson Imaging. 2002;15(3):302-7. [PubMed: 11891975].

9. Oka K, Yakushiji T, Sato H, Yorimitsu S, Hayashida Y, Yamashita $\mathrm{Y}$, et al. Ability of diffusion-weighted imaging for the differential diagnosis between chronic expanding hematomas and malignant soft tissue tumors. J Magn Reson Imaging. 2008;28(5):1195-200. doi: 10.1002/jmri.21512. [PubMed: 18972359]. 